Vol. 43 (1991) [265-267]

\title{
A CHARACTERISATION RESULT FOR MATRIX RINGS
}

\author{
P.R. FuChS
}

For a ring $R$ with identity we show that the existence of certain nilpotent elements forces $R$ to be a matrix ring of size $\geqslant 2$.

In structure theory it is often useful to know whether a given ring $R$ with identity is isomorphic to a matrix ring over some ring $S$ with a more tractable structure. For instance, it is nice to have that $S$ is an integral domain.

A ring $R$ is isomorphic to a matrix ring of size $n$ if and only if there exists a set of matrix units $\left\{e_{i j} \mid 1 \leqslant i, j \leqslant n\right\} \subseteq R$, that is, $\sum_{i=1}^{n} e_{i i}=1$ and $e_{i j} e_{k l}=\delta_{j k} e_{i l}$ where $\delta_{j k}$ is the Kronecker delta. In this case $R \cong M_{n}(S)$, where $S \cong e_{i i} R e_{i i}$ for all $1 \leqslant i \leqslant n$. For this and other well-known facts concerning matrix rings see [2]. In this note we prove the following criterion for $R$ to be a matrix ring. Ann ( $s)$ shall denote the left annihilator of an element $s \in R$.

Theorem 1. For a ring $R$ with identity 1 the following are equivalent:

(1) $R \cong M_{n}(S)$ for some ring $S$ and some positive integer $n \geqslant 2$.

(2) For some positive integer $n \geqslant 2$, there exist elements $x, y \in R$ such that $x^{n-1} \neq 0, x^{n}=y^{2}=0, x+y$ is invertible and Ann $\left(x^{n-1}\right) \cap R y=(0)$.

Moreover if (2) holds and $r$ is the inverse of $x+y$, then $\left\{e_{i j} \mid 1 \leqslant i, j \leqslant n\right\}$, where $e_{i j}=r^{n-i}(r y) x^{n-j}$, is a set of matrix units for $R$. Thus, if $e$ denotes the idempotent $r y=e_{n n}$, then $R \cong M_{n}(S)$, where $S \cong e R e$.

Condition 2 in Theorem 1 can often be easily verified or rejected. Also, once the inverse $r$ of $x+y$ is known, the matrix units for $R$ are given explicitly. For the proof of Theorem 1 we need two propositions in which we keep the notation of Theorem 1 and assume that condition 2 holds.

PROPOSITION 2. $y r^{k} y=0$ for all $2 \leqslant k \leqslant n$.

PROOF: Since $r x+r y=1, y=y r x+y r y$, hence $y r x \in R x \cap R y$. But $R x \subseteq$ Ann $\left(x^{n-1}\right)$, so $R x \cap R y=(0)$, that is $y r x=0$. Since $x^{n-1}=r y x^{n-1}, y r^{2} y x^{n-1}=$ $y r x^{n-1}=0$; thus $y r^{2} y \in \operatorname{Ann}\left(x^{n-1}\right) \cap R y=(0)$. Consequently $y r=y r^{2} x+y r^{2} y=$

Received 20 April 1990

Copyright Clearance Centre, Inc. Serial-fee code: 0004-9729/91 \$A2.00+0.00. 
$y r^{2} x$, and hence $y r^{2} x^{2}=y r x=0$. Now let $2<k \leqslant n$ and suppose we have shown that $y r^{i} y=0, y r^{i} x^{j}=0$ for all $2 \leqslant i<k$ and $i \leqslant j$. Then $y r^{k} y x^{n-1}=y r^{k-1} x^{n-1}=0$; hence $y r^{k} y=0$. Thus $y r^{k-1}=y r^{k} x+y r^{k} y=y r^{k} x$. By our induction hypothesis $y r^{k-1} x^{j}=0$ for all $j \geqslant k-1$; hence $y r^{k} x^{j}=0$ for all $j \geqslant k$. Our claim now follows.

Proposition 3. $r y x^{i} r^{j} r y=\delta_{i j} r y$ for all $0 \leqslant i, j \leqslant n-1$.

Proof: Let $0 \leqslant i \leqslant n-1$. We show that $r y x^{i} r^{i} r y=r y$. In Proposition 2 we have seen that $y r x=0$; hence $y=y r y$, that is, $r y$ is idempotent. So our claim is true for $i=0$. Since $x r+y r=1$, we have $x^{i-1} r^{i-1}=x^{i} r^{i}+x^{i-1} y r r^{i-1}$ for $1 \leqslant i \leqslant n-1$, and hence $x^{i-1} r^{i-1} r y=x^{i} r^{i} r y+x^{i-1} y r^{i+1} y$. Since $i+1 \leqslant n, y r^{i+1} y=0$ by Proposition 2 , thus $x^{i-1} r^{i-1} r y=x^{i} r^{i} r y$. Consequently $x^{i} r^{i} r y=r y$ for all $0 \leqslant i \leqslant n-1$.

Now let $i \neq j$. If $i>j$, then $x^{i} r^{j} r y=x^{i-j} x^{j} r^{j} r y=x^{i-j} r y$. But since $x=$ $x r x+x r y, x r y \in R x \cap R y=(0)$. Finally suppose that $i<j$. If $i=0$, then $r y x^{i} r^{j} r y=r y r^{j+1} y=0$, since $j+1 \leqslant n$.

Now let $i>0$ and $0<k \leqslant i$. Then $x^{i-k} r^{i-k} r^{j-i} r y=x^{i-k+1} r^{i-k+1} r^{j-i} r y+$ $x^{i-k} y r r^{i-k} r^{j-i} r y$. But since $k>0$ and $j \leqslant n-1, j-k+2 \leqslant n$; hence $y r^{j-k+2} y=0$ and therefore $x^{i-k} r^{i-k} r^{j-i} r y=x^{i-k+1} r^{i-k+1} r^{j-i} r y$. It now follows that $x^{i} r^{j} r y=$ $x^{i} r^{i} r^{j-i} r y=r^{j-i} r y$, and thus $r y x^{i} r^{j} r y=r y r^{j-i+1} y=0$.

We are now ready to prove Theorem 1 .

Proof of Theorem 1: $2 \Rightarrow 1$. For $1 \leqslant i, j \leqslant n$ let $e_{i j}=r^{n-i}(r y) x^{n-j}$. It is then immediate from Proposition 3 that $e_{i j} e_{k l}=\delta_{j k} e_{i l}$. It remains to show that $\sum_{i=1}^{n} e_{i i}=1$. Since $r x+r y=1, r^{n-i} x^{n-i}=r^{n-i+1} x^{n-i+1}+r^{n-i}(r y) x^{n-i}$ for all $1 \leqslant i \leqslant n-1$. But $r^{n} x^{n}=0$, thus $\sum_{i=1}^{n-1} r^{n-i}(r y) x^{n-i}=\sum_{i=1}^{n-1} e_{i i}=r x$. Since $r y=e_{n n}$, $\sum_{i=1}^{n} e_{i i}=1$.

$1 \Rightarrow 2$. Let $\left\{e_{i j} \mid 1 \leqslant i, j \leqslant n\right\}$ be a set of matrix units for $R$. If $x:=$ $e_{12}+\ldots+e_{n-1 n}, y:=e_{n 1}$, then $x^{n-1} \neq 0, x^{n}=y^{2}=0$ and $x+y$ is invertible with inverse $r=e_{1 n}+e_{21}+\ldots+e_{n n-1}$. Moreover one checks that $\operatorname{Ann}\left(x^{n-1}\right)=R x$ and that $R x \cap R y=(0)$.

The following special case of Theorem 1 has also been stated in [1] (Theorem III.2).

COROLlary 4. For a ring $R$ with identity the following are equivalent:

(1) $R$ is a ring of $2 \times 2$ matrices over some ring $S$.

(2) There exist elements $x, y \in R$ such that $x^{2}=y^{2}=0$ and $x+y$ is invertible.

Corollary 4 follows immediately from Theorem 1 for if $0 \neq s y \in \operatorname{Ann}(x) \cap R y$, then $s y \in \operatorname{Ann}(x+y)$ which is a contradiction to the fact that $x+y$ is invertible. 
In a forthcoming paper we give some applications of Theorem 1 in structure theory. For instance, if $R$ is a prime Goldie ring we show how elements $x, y$ satisfying condition 2 can be constructed in the quotient ring of $R$. In this manner we obtain another proof for the fact that the quotient ring of $R$ is isomorphic to a matrix ring over a division ring.

\section{REFERENCES}

[1] P.R. Fuchs, C.J. Maxson and G. Pilz, 'On rings for which homogeneous maps are linear', Proc. Amer. Math. Soc (to appear).

[2] L. Rowen, Ring theory, Vol. 1 (Academic Press, 1988).

Institut für Mathematik

Johannes Kepler Universitāt

A-4040 Linz

Austria 